\title{
Advances in endoscopic imaging: Advantages and limitations
}

\author{
Rupa Banerjee, D. Nageshwar Reddy \\ Department of Medical Gastroenterology, Asian Institute of Gastroenterology, Hyderabad, India
}

\begin{abstract}
\end{abstract}
\section{Introduction}

Gastrointestinal endoscopy revolutionized the diagnosis and therapy of many gastrointestinal diseases in the last two decades. Endoscopic imaging is again undergoing a profound evolutionary change. The perspective of diagnostic endoscopy is changing from diagnosing evident disease to the detection of subtle abnormalities.

Conventional white light endoscopy (WLE) has been limited to detecting lesions on the basis of gross morphological changes. Diagnosis is primarily based on biopsy sampling of obvious macroscopic endoscopic features, or "blind" biopsy sampling of normal appearing mucosa. These random biopsies are clearly inefficient and miss significant histology. WLE is often unable to identify subtle mucosal alteration in flat lesions which prevents early detection of GI malignancies in the resectable and curative stages.

The newer endoscopic imaging technologies attempt to

\begin{tabular}{|l|c|}
\hline \multicolumn{2}{|c|}{ Access this article online } \\
\hline \multirow{2}{*}{$\begin{array}{l}\text { Website: } \\
\text { www.jdeonline.in }\end{array}$} & Quick Response Code \\
\hline DOI: & \\
10.4103/0976-5042.95023 & \\
\hline
\end{tabular}

visualize what was formerly possible only with biopsy and histologic interpretation. High resolution, high definition systems have CCDs of more than a million pixels which are 3 times higher than the conventional scopes. It focuses on early detection and categorization and optimization of treatment.

The aims of advanced imaging beyond WLE include:

a) Improved detection of minute lesions and mucosal alterations with a potential for neoplasia.

b) To provide characterization of the tissue of interest neoplastic/nonneoplastic or inflammatory.

c) In vivo histology and optical biopsy.

These advances can be categorized into:

a) Image enhanced endoscopy or field enhancement as a red flag technology to survey the entire lumen of the gastrointestinal tract. This encompasses contrast enhancement using dye, optical, and/or electronic methods and include Chromoendoscopy, NBI, I scan, FICE and autofluorescence endoscopy.

b) Virtual histology or point enhancement for in vivo hislogical examination during endoscopy. Confocal laser endomicroscopy, endocytoscopy and OCT provide cellular and subcellular imaging for real time optical biopsy.

This review attempts to define the current status of these newer technologies and the advantages and limitations in current clinical practice. 


\section{Chromoendoscopy}

Chromoendoscopy involves the application of stains or dyes to the gastrointestinal mucosa during endoscopy to improve tissue visualization, characterization and diagnosis by enhancing the contrast. ${ }^{[1]}$

The stain/dye solutions are classified into:

a) Vital stains which are taken up by specific epithelial cells. Eg Lugols iodine, methylene blue, toluidine blue and crystal violet.

b) Contrast stains which are not absorbed but pool up in the crevices of the mucosa and highlight the fine mucosal irregularities. Eg Indigo carmine.

c) Reactive stains which undergo color change based on the presence of a alkaline/acidic $\mathrm{pH}$. e.g. Congo red, phenol red.

\section{Chromoendoscopy has been mostly studied for}

i. Detection of dysplasia and early carcinomas of stomach and esophagus. Lugols iodine most commonly used. It also improves the visualization of the lateral margins of the lesion and delineates the extent.

ii. Detection and characterization of polyps. ${ }^{[2-5]}$ Indigo carmine has been used most commonly. The sensitivity and specificity of differentiation ranges from $82-98 \%$ and $52-95 \%$ respectively.

iii. Detection of dysplasia in chronic ulcerative colitis. ${ }^{[-8]}$

Chromoendoscopy is considered a simple, safe and inexpensive technique that is useful in identifying premalignant conditions and minute cancerous lesions as well as predicting the histological type and submucosal invasion. Some limitations have prevented the widespread use of chromoendoscopy in clinical practice beyond few specified conditions. These include the lack of standardized classification systems for chromoendoscopic findings and poor reproducibility. Also, the routine use of these dyes can be messy and time consuming and the cost effectiveness is yet undefined.

\section{Digital chromoendoscopy}

These are novel optical technologies that enhance surface pit pattern and microvasculature without the use of dyes. These include

a) Narrow band imaging (NBI), b) I scan and c) FICE

\section{Narrow band imaging}

This is the most widely used advanced imaging technology primarily because of the ease of usage and availability at the switch of a button. ${ }^{[9]} \mathrm{NBI}$ technology involves the placement of narrow band pass filters in front of a conventional white light source to obtain tissue illumination at selected narrow wavelength bands. This provides a real time on demand optical image enhancement that enhances visualization of the vascular network and surface texture of the mucosa thereby assisting in tissue characterization, differentiation and diagnosis. ${ }^{[9,10]}$ The NBI system components are identical to the conventional RGB sequential or color CCD endoscopes. The primary modifications are within the light source where an optical filter with narrow band transmission is placed. NBI systems have also been coupled with electronic or optical zoom facilities for enhanced visualization of mucosal details. The commercially available NBI systems include the 2-band NBI RGB sequential endoscopes (Evis Lucera 260 Spectrum) and the color CCD endoscopes (Evis Exera II 180, Olympus Medical Systems, Tokyo, Japan).

\section{Current role of Narrow band imaging in clinical practice}

NBI has primarily been applied in the analysis of the surface architecture of the epithelium (pit pattern) and the analysis of the vascular network. It can demonstrate and distinguish the alteration in the pit pattern and vasculature between inflammatory and neoplastic lesions of the esophagus, stomach and large bowel. ${ }^{[1]]}$

Currently available literature supports the use of NBI for surveillance in Barrett's oesophagus and in patients considered at high risk for squamous cancers of the oropharynx, oesophagus and stomach. ${ }^{[9,10,12,13]}$ NBI targeted biopsy can enhance dysplasia detection compared to WLE in the surveillance of inflammatory bowel disease ${ }^{[14]}$ Emerging data also supports the use of NBI in therapeutic procedures including ablation of Barrett's, endoscopic mucosal resection and ESD where the assessment of the margin is critical. However interpretation of contrast enhanced images require familiarity and may not be straight forward with considerable inter observer variation. The presence of blood or bile prevents optimal viewing.

NBI is an emerging technology. The classifications of various conditions described on NBI need to be further standardized and validated for use in routine clinical practice.

\section{I scan/Fuji Intelligent Chromo Endoscopy}

I scan from Pentax (Montvale, NJ) ${ }^{[15]}$ and Fuji Intelligent Chromo Endoscopy (FICE) (Fujinon, Wayne, NJ) are based on post imaging processing and involve spectral estimation technology. ${ }^{[16]}$ This involves taking an ordinary endoscopic image from the video processor and production of an image of a given, dedicated wavelength of light after arithmetic estimation. There is no optical filter involved in contrast to narrow band imaging.

Only a limited number of studies for these technologies have been reported. These have reported has enhanced adenoma detection rates and differentiation of neoplastic and non-neoplastic colorectal lesions compared to WLE. ${ }^{[17]}$ Head to head comparisons between these technologies are not available.

\section{Autofluorescence endoscopy}

Autofluorescence imaging (AFI) is based on the detection of the relative concentration of endogenous fluorophores and fluorescence emission thereby allowing differentiation between healthy and neoplastic tissue. AFI endoscopy provides real time images from the CCD computerization of captured fluorescence based on the same principles. The endogenous 
tissue fluorophores of the gastrointestinal tract include tyrosine, tryptophan, NADH, FAD, elastin, collagen and porphyrins. These fluorophores are excited on exposure to short wavelength light leading to the emission of a fluorescent light of a longer wavelength (autofluorescence). ${ }^{[18]}$ The autofluorescence characteristics of normal, metaplastic and dysplastic epithelial structures are dependent on the type, concentration and distribution of different fluorophores together with the biochemical composition and perfusion characteristics. ${ }^{[19]}$ Malignant transformation of tissue is associated with emission of relatively longer wavelengths of light. The initial AFI endoscopy systems used fiber optic technology and hence the quality of images was poor. ${ }^{[20,21]}$ The more recent video systems have substantially improved the quality and resolution of the images. Dysplastic lesions are displayed in a brilliant magenta color against a green/cyancoloured normal background. Non neoplastic tissues appear green.

The primary goal of this wide area functional imaging is as a red flag technology to rapidly examine a large surface of gastrointestinal mucosa for early identification of a possible dysplastic area and image guided targeted biopsy.

Several controlled trials have shown the superiority of AFI over white light endoscopy in detecting high grade dysplasia in Barrett's esophagus, superficial esophageal squamous cell carcinoma and early gastric cancer. AFI colonoscopy has been useful in the distinction between adenomatous and hyperplastic polyps. Uedo and colleagues found that AFI endoscopy accurately identified the extent of superficial oesophageal carcinoma in 5 of 5 patients and gastric carcinoma in 15 of $22(68 \%)$ whereas WLE detected only 2 of 5 and $22 \%$ cases respectively. ${ }^{[22]}$ Kara et al. similarly increased detection rates of high grade dysplasia in Barrett's oesophagus from 22\% to $33 \%$. $^{[23]}$

However there are still too many false positives to make AFI useful as a standalone technique in clinical practice. The image quality though improved is still poorer than the high definition systems. Subsurface changes cannot be identified. Also the color tone is unstable related to the presence of mucus, instrument angulation and air insufflation.

Summarily AFI is primarily a wide area imaging technology which can be used as a red flag. The rate of false positives is however high and further improvements in fluorescence image quality is required. Trimodal imaging appears promising and larger validation studies are warranted.

\section{Trimodal imaging}

False positivity is the major drawback of AFI. To decrease false positivity AFI has been combined with high resolution WLE and NBI (Trimodal imaging). ${ }^{[2]}$

A novel prototype trimodal endoscope is available which incorporates high resolution WLE, NBI and autofluorescence. In a multicenter study on 84 patients with Barrett's oesophagus
AFI increased the detection rate from 16 of 30 on WLE to 27 of 30 . AFI also increased the detection rate of biopsy proven lesions from 21 with WLE to 40 with AFI. However false positive rates was high $(81 \%)$. NBI reduced the false positivity to $26 \%$. ${ }^{[25]}$

\section{Optical biopsy: In vivo histologic assessment}

Recent advances in endoscopic imaging technology have now made microscopic observation possible at the cellular level assisting in tissue characterization of a variety of neoplastic and non neoplastic lesions of the gastrointestinal tract. These include i) Endocytoscopy (EC) ii) Confocal laser endomicroscopy (CLE) iii) Optical Coherence Tomography (OCT)

\section{Endocytoscopy}

The Endocytoscope (E-C) system is based on the principle of light contact microscopy and enables on the spot assessment for cellular atypia. It has been primarily been used for the in vivo assessment of the oesophageal mucosa. ${ }^{[26]}$

The prototype EC system (Olympus Corporation, Tokyo, Japan) consists of two flexible endoscopes (3.2 mm diameter) each that can easily pass through the accessory channel of any endoscope with a working channel of $3.7 \mathrm{~mm}$. A prototype unified NBI system integrated with EC facilities is also under evaluation.

The endocytoscope is passed through the accessory channel of a regular endoscope or colonoscope and the tip placed in direct contact with the surface after application of $1 \%$ methylene blue. ${ }^{[27]}$

The normal cells are arranged homogenously with a normal nuclear cytoplasmic ratio. Mitotic cells on the other hand appear heterogenous in shape with altered nuclear cytoplasmic ratio and are arranged in irregular clusters.

The EC system has been used successfully in detection of mitotic changes in vivo in oesophageal carcinoma. ${ }^{[26]}$ Preliminary reports show good correlation with histology. We have recently reported the use of intra-operative endocytoscopy in detecting pancreatic head carcinoma in a patient with chronic pancreatitis. The targeted biopsies correlated with the frozen section and conventional histopathology.

There are key challenges before endocytoscopy can be accepted into routine clinical practice. The presence of mucus or blood may obscure view. Respiratory and cardiac movements can also hamper visualization. It is difficult to map the exact area of involvement since the whole area cannot be observed at the same time.

\section{Confocal laser endomicroscopy}

Confocal endomicroscopy (CEM) provides images from layers of tissue using the principle of optical sectioning at the cellular and subcellular structures after the topical/IV application of 
exogenous fluorescence contrast agents including fluorescein, acriflavin or cresyl violet. ${ }^{[28]} \mathrm{CEM}$ is an accepted standard method for localization of specific proteins of cellular structures in basic research. Endoscopically this new imaging modality not only provides conventional histology but also indicates the pathophysiology as cellular interaction can be observed over time. The current CLE incorporates a confocal laser microscope into the tip of a flexible endoscope (Pentax EC 3830FK, Tokyo, Japan) allowing a 1000-fold magnification with high resolution and real time in vivo histology of the gastrointestinal tract mucosa. A probe based confocal endomicroscope (Cellvizio, Mauna Kea technologies, France) is also available. These confocal miniprobes can be passed over the working channel of all standard endoscopes. ${ }^{[29]}$ An ERCP dedicated mini probe (Cholangioflex) for the cholangioscope allows real time microscopic level visualization of the bile and pancreatic ducts.

Clinical applications of CLE have aimed at the prediction of gastrointestinal neoplasias and the generation of targeted biopsies. The cellular information obtained by pCLE has been used to target tissue sampling (brushings, biopsies or fine needle aspiration) and to assist in making treatment decisions on the spot.

Preliminary results in detection of intraepithelial neoplasias in Barrett's esophagus, ulcerative colitis surveillance, differentiation of nature of colorectal polyps and characterization of CBD strictures have been encouraging. ${ }^{[30]}$

CLE adds a new dimension to the endoscopic armamentarium in gastroenterology and is likely to have great impact on gastrointestinal endoscopy in the future. However, this technology is not suited for screening of large surfaces as during UC surveillance. Additionally, it is an examiner dependent technology and training on interpretation is of crucial importance before reliable histologic diagnoses can be made.

\section{Optical coherence tomography}

OCT Optical coherence tomography (OCT) is an optical imaging modality that performs high-resolution, crosssectional, subsurface tomographic imaging of the microstructure of tissues. The physical principle of OCT is similar to that of B-mode ultrasound imaging, except that it uses infrared light waves rather than acoustic waves. OCT can be done by using narrowdiameter, catheter-based probes that can be inserted through the accessory channel of an endoscope. ${ }^{[31]}$

OCT imaging of the gastrointestinal wall is characterized by a multiple layer architecture that permits an accurate evaluation of the mucosa, lamina propria, muscularis mucosae, and part of the submucosa. The technique has been used to identify pre-neoplastic conditions, such as Barrett's epithelium and dysplasia, and evaluate the depth of penetration of early neoplastic lesions of the GI and pancreatobiliary system.
A few preliminary studies involving small number of patients are currently available. Larger carefully conducted prospective trials are necessary to determine the clinical utility of this imaging modality.

\section{Imaging modalities in the pipeline Fluorescence, light scattering and Raman spectroscopy}

These are optical spectroscopic techniques based on the analysis of specific light -tissue interactions such as fluorescence, elastic scattering and inelastic (Raman) scattering. ${ }^{[32,33]}$ Spectral differences in the optical signals based on the microstructure and biochemical nature of the tissues can differentiate between neoplastic and non neoplastic tissues. Several proof of concept studies have demonstrated the usefulness of spectroscopy for the optical detection of dysplasia in Barretts and early gastric carcinomas and the differentiation of colorectal polyps. Future technologic developments and the development of highly selective fluorophores as biomarkers will enhance diagnostic accuracy of spectroscopic imaging.

\section{Reflectance spectroscopy}

Reflectance spectroscopy is a point probe optically based endoscopic technique that quantitatively measures the color and intensity of reflected light. It thereby provides information about tissue haemoglobin concentrations and oxygenation status. The inherent property of malignant tissue to promote angiogenesis enables differentiation between mitotic and benign tissues.

\section{Targeted endoscopic imaging}

This attempts to acquire real time data about the molecular expression of cells and tissues within the digestive tract. Changes in molecular expression occur well before that of structural features and fluorescence/CLE imaging of these expressed targets can enable the earliest detection of cancer as well as response to therapy. The best studied intracellular targets are proteolytic enzymes and surface targets transmembrane proteins and glycoproteins.

This is the upcoming technology for the future management of patients who have an increased likelihood of cancer including risk stratification, early detection, therapeutic monitoring and evaluation of recurrence.

\section{The future of advanced imaging: Multimodal imaging}

Different modalities can be merged into new multimodal devices making it possible to measure multiple parameters in one procedure. This also paves the way for fusion of diagnostic and therapeutic imaging enabling patients to have a one-session procedure. Multimodal imaging can in fact utilize the strengths of each individual technique and minimize the limitations and thereby enhance visualization of not only mucosal surface and microvasculature but also the cellular and subcellular architecture. 
A proposed algorithm for enhanced multimodal imaging is depicted below Table 1 .

A novel trimodal imaging system has recently become available that incorporates high-resolution endoscopy, AFI and NBI in one single system: Known as endoscopic tri-modality imaging (ETMI). AFI alone has a high false positive rate but with ETMI characterization the false positive rate dropped down. Trimodal imaging can actually serve as a 'red flag' technique for the detection of early neoplastic lesions by AFL followed
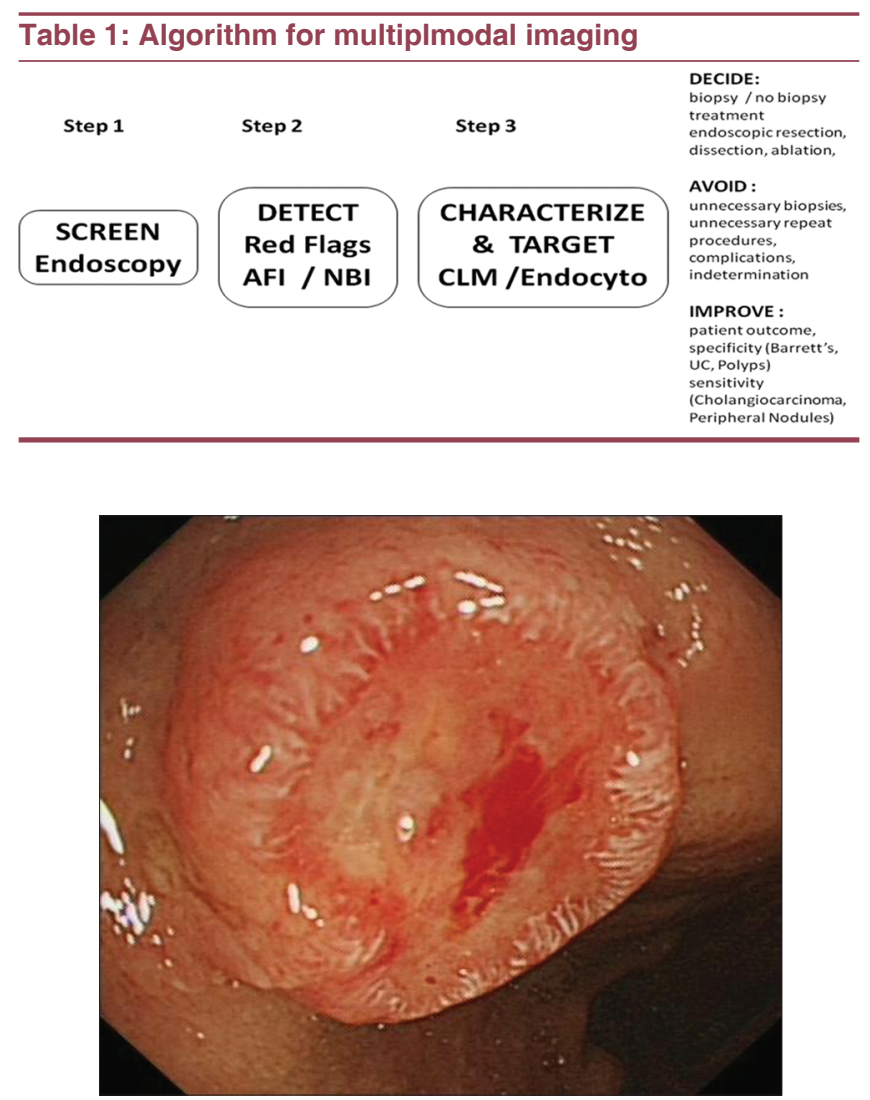

Figure 1a: WLE showing a well demarcated ulcer with raised margins in the duodenum by NBI to verify surface pattern characteristics.

Trimodal imaging has been reported to be effective in screening for early neoplasia in Barretts oesophagus and dysplasia in ulcerative colitis. We have used the ETMI for differentiation between adenomatous and hyperplastic polyps as well as between neoplastic and non neoplastic lesions with good concordance with histopathology [Figures 1a - c].

\section{Conclusion}

Gastrointestinal cancers are a leading cause of cancer-related death worldwide. The best chances of cure are associated with early diagnosis. However these precancerous changes are often subtle and cannot be identified during standard white light endoscopy. Recent advances in imaging allow better detection of these early neoplastic lesions and increase the effectiveness of endoscopic surveillance and screening.

These tools have a potential to improve with further technologic improvement. Future applications may include

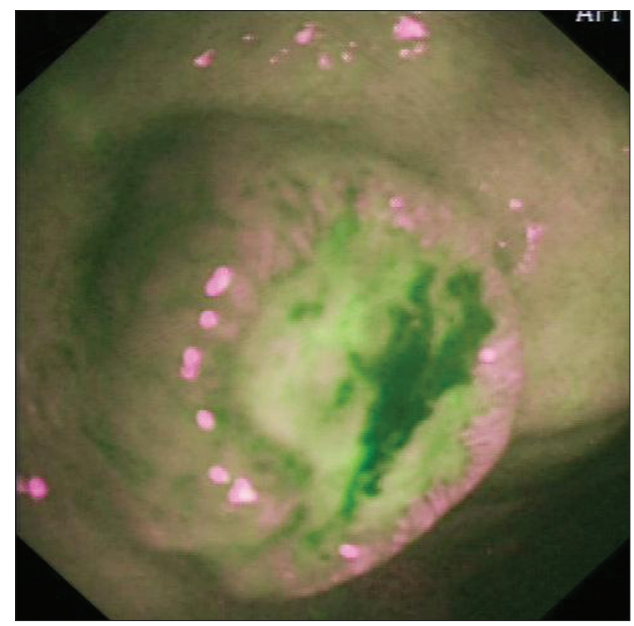

Figure 1b: Distinct magenta hue at the margins on AFI
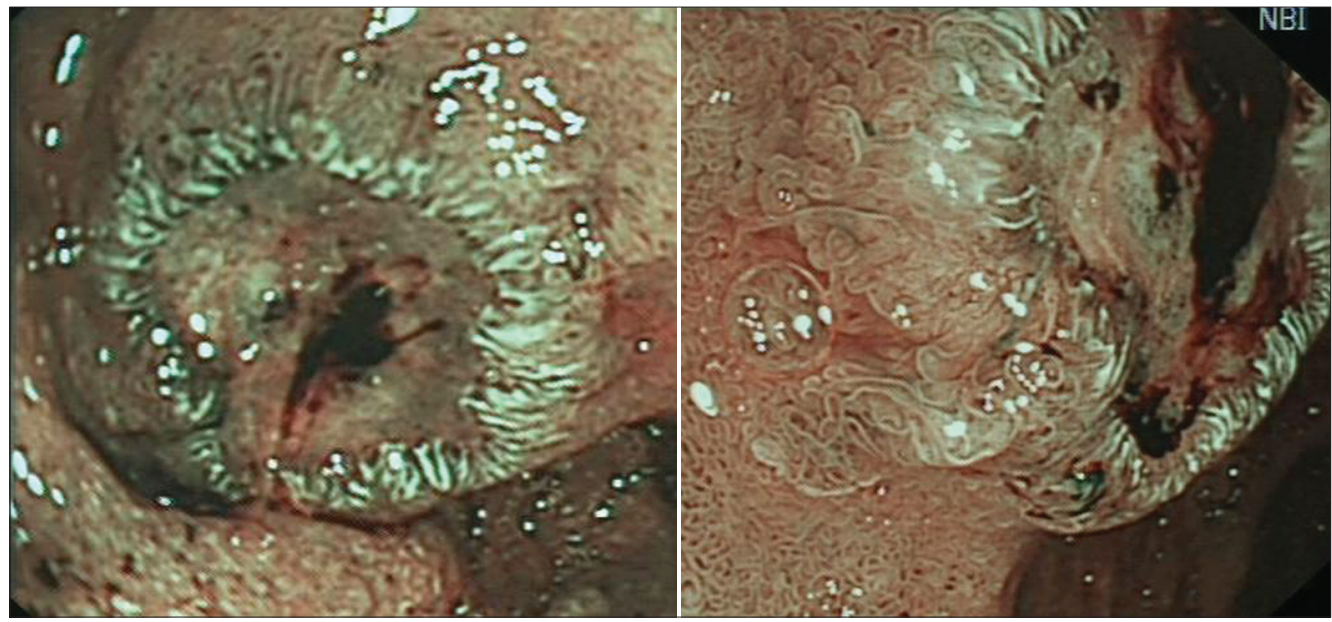

Figure 1c: Characteristic light blue crest on NBI. Inset shows irregular pit pattern at the periphery. Biopsy suggestive of neuroendocrine tumour 
targeted detection of intracellular and surface targets expressed by the mucosa using fluorescence or confocal endomicroscopy even prior to the development of cancer. However, there are many challenges ahead. As with all emerging newer technologies, the limitations include lack of standardized classification systems and poor reproducibility. Personal skills and experiences affect detection and diagnostic ability. Systematic additional training may be required before endoscopists can interpret cellular and subcellular images. The cost effectiveness and ultimate impact on patient care also needs to be evaluated before incorporation of these technologies into routine clinical practice.

\section{Reference}

1. Wong KeeSong LM, Adler DG, Chand B, ConwayJD, Croffie JM, Disario JA, et al. Chromoendoscopy. Gastrointest. Endosc. 2007;66:639-49.

2. Brooker JC, Saunders BP, Shah SG, Thapar CJ, Thomas HJ, Atkin WS, et al. Total colonic dye-spray increases the detection of diminutive adenomas during routine colonoscopy: A randomized controlled trial. Gastrointest. Endosc. 2002;56:333-38.

3. Lee JH, Kim JW, Cho YK, Sohn CI, Jeon WK, Kim BI, et al. Detection of colorectal adenomas by routine chromoendoscopy with indigocarmine. Am J Gastroenterol 2003;98:1284-8.

4. Eisen GM, Kim CY, Fleischer DE, Kozarek RA, Carr-Locke DL, Li TC, et al. High-resolution chromoendoscopy for classifying colonic polyps: A multicenter study. Gastrointest Endosc 2002;55:687-94.

5. Fu KI, Sano Y, Kato S, Fujii T, Nagashima F, Yoshino T, et al. Chromoendoscopy using indigo carmine dye spraying with magnifying observation is the most reliable method for differential diagnosis between non-neoplastic and neoplastic colorectal lesions: A prospective study. Endoscopy 2004;36:1089-93.

6. Hurlstone DP, Sanders DS, Lobo AJ, McAlindon ME, Cross SS. Indigo carmine-assisted high-magnification chromoscopic colonoscopy for the detection and characterisation of intraepithelial neoplasia in ulcerative colitis: A prospective evaluation. Endoscopy 2005;37:1186-92.

7. Kiesslich R, Fritsch J, Holtmann M, Koehler HH, Stolte M, Kanzler S, et al. Methylene blue-aided chromoendoscopy for the detection of intraepithelial neoplasia and colon cancer in ulcerative colitis. Gastroenterology 2003;124:880-8.

8. Rutter MD, Saunders BP, Schofield G, Forbes A, Price AB, Talbot IC. Pancolonic indigo carmine dye spraying for the detection of dysplasia in ulcerative colitis. Gut 2004;53:256-60.

9. Sharma P, Bansal A, Mathur S, Wani S, Cherian R, McGregor D, et al. The utility of a novel narrow band imaging endoscopy system in patients with Barrett's esophagus. Gastrointest. Endosc. 2006;64:167-75.

10. Reddymasu SC, Sharma P. Advances in endoscopic imaging of the esophagus. Gastroenterol Clin North Am 2008;37:763-74.

11. Banerjee R, Reddy DN. Enhanced endoscopic imaging and gastroesophageal reflux disease. Indian J Gastroenterol 2011;30:193-200.

12. Rastogi A, Bansal A, Wani S, Callahan P, McGregor DH, Cherian R, et al. Narrow-band imaging colonoscopy-a pilot feasibility study for the detection of polyps and correlation of surface patterns with polyp histologic diagnosis. Gastrointest Endosc 2008;67:280-6.

13. Sharma P, Wani S, Bansal A, Hall S, Puli S, Mathur S, et al. A feasibility trial of narrow band imaging endoscopy in patients with gastroesophageal reflux disease. Gastroenterology 2007;133:454-44.

14. Nass JP, Connolly SE. Current status of chromoendoscopy and narrow band imaging in colonoscopy. Clin Colon Rectal Surg 2010;23:21-30.

15. Lee CK, Lee SH, Hwangbo Y. Narrow-band imaging versus I-Scan for the real-time histological prediction of diminutive colonic polyps: A prospective comparative study by using the simple unified endoscopic classification. Gastrointest Endosc 2011;74:603-9.

16. Jung SW, Lim KS, Lim JU, Jeon JW, Shin HP, Kim SH, et al. Flexible spectral imaging color enhancement (FICE) is useful to discriminate among non-neoplastic lesion, adenoma, and cancer of stomach. Dig. Dis. Sci. 2011;56:2879-86.

17. Yoshida N, Naito $\mathrm{Y}$, Inada $\mathrm{Y}$, Kugai $\mathrm{M}$, Inoue $\mathrm{K}$, Uchiyama $\mathrm{K}$, et al. The detection of surface patterns by flexible spectral imaging color enhancement without magnification for diagnosis of colorectal polyps. Int J Colorectal Dis. 2011 Dec 6. [Epub ahead of print].

18. Kara MA, DaCosta RS, Streutker CJ, Marcon NE, Bergman JJ, Wilson BC. Characterization of tissue autofluorescence in Barrett's esophagus by confocal fluorescence microscopy. Dis Esophagus 2007;20:141-50.

19. Kara MA, Bergman JJ. Autofluorescence imaging and narrow-band imaging for the detection of early neoplasia in patients with Barrett's esophagus. Endoscopy 2006;38:627-31.

20. Haringsma J, Tytgat GN, Yano H, Iishi H, Tatsuta M, Ogihara T, et al. Autofluorescence endoscopy: Feasibility of detection of GI neoplasms unapparent to white light endoscopy with an evolving technology. Gastrointest Endosc 2001;53:642-50.

21. Niepsuj K, Niepsuj G, Cebula W, Zieleźnik W, Adamek M, Sielańczyk A, et al. Autofluorescence endoscopy for detection of high-grade dysplasia in short-segment Barrett's esophagus. Gastrointest Endosc 2003;58:715-9.

22. Uedo N, Iishi H, Tatsuta M, Yamada T, Ogiyama H, Imanaka $\mathrm{K}$, et al. A novel videoendoscopy system by using autofluorescence and reflectance imaging for diagnosis of esophagogastric cancers. Gastrointest Endosc 2005;62:521-8.

23. Kara MA, Peters FP, Ten Kate FJ, Van Deventer SJ, Fockens P, Bergman JJ. Endoscopic video autofluorescence imaging may improve the detection of early neoplasia in patients with Barrett's esophagus. Gastrointest Endosc 2005;61:679-85.

24. Rotondano G, Bianco MA, Sansone S, Prisco A, Meucci C, Garofano ML, et al. Trimodal endoscopic imaging for the detection and differentiation of colorectal adenomas: A prospective single-centre clinical evaluation. Int J Colorectal Dis 2012;27:331-6.

25. Curvers WL, Herrero LA, Wallace MB, Wong Kee Song LM, Ragunath K, Wolfsen HC, et al. Endoscopic tri-modal imaging is more effective than standard endoscopy in identifying early-stage neoplasia in Barrett's esophagus. Gastroenterology 2010;139:1106-14.

26. Banerjee R, Reddy DN, Rao GV, Shekharan A, Ramji C. Application of high-resolution narrow band imaging and endocytoscopy for early diagnosis of esophageal neoplasia. Indian J Gastroenterol 2008;27:204-6.

27. Neumann H, Fuchs FS, Vieth M, Atreya R, Siebler J, Kiesslich R, et al. Review article: In vivo imaging by endocytoscopy. Aliment Pharmacol Ther 2011;33:1183-93.

28. Kiesslich R, Burg J, Vieth M, Gnaendiger J, Enders M, Delaney P, et al. Confocal laser endoscopy for diagnosing intraepithelial neoplasias and colorectal cancer in vivo. Gastroenterology 2004;127:706-13.

29. Wallace MB, Sharma P, Lightdale C, Wolfsen H, Coron E, Buchner A, et al. Preliminary accuracy and interobserver agreement for the detection of intraepithelial neoplasia in Barrett's esophagus with probe-based confocal laser endomicroscopy. Gastrointest Endosc 2010;72:19-24.

30. Sharma P, Meining AR, Coron E, Lightdale CJ, Wolfsen HC, Bansal A, et al. Real-time increased detection of neoplastic tissue in Barrett's esophagus with probe-based confocal laser endomicroscopy: Final results of an international multicenter, prospective, randomized, controlled trial. Gastrointest Endosc 2011;74:465-72.

31. Thekkek N, Anandasabapathy S, Richards-Kortum R. Optical molecular imaging for detection of Barrett's-associated neoplasia. World J Gastroenterol 2011;17:53-62.

32. Li M, Anastassiades CP, Joshi B, Komarck CM, Piraka C, Elmunzer BJ, et al. Affinity peptide for targeted detection of dysplasia in Barrett's esophagus. Gastroenterology 2010;139:1472-80.

33. Wallace MB, Wax A, Roberts DN, Graf RN. Reflectance spectroscopy. Gastrointest. Endosc. Clin. N. Am. 2009;19:233-42.

How to cite this article: Banerjee R, Reddy DN. Advances in endoscopic imaging: Advantages and limitations. J Dig Endosc 2012;3:7-12.

Source of Support: Nil, Conflict of Interest: None declared. 